\title{
LA CABEZA DE JANO EN LA EVOLUCIÓN DEL IUS IN BELLO INTERNO Y LA CUESTIÓN DEL NUEVO IUS CONTRA BELLUM INTERNUM
}

Klaus Kress*

Resumen: Desde 1945, la mayoría de los conflictos armados tuvieron lugar entre Estados y rebeldes o entre grupos armados no estatales al interior de Estados frágiles o fallidos. En la actualidad, los dramas en Irak, Siria y en Ucrania nos muestran que la guerra civil es uno de los males más importantes de nuestro tiempo. Reconociendo este hecho, el Derecho del conflicto armado no internacional, en particular desde la primera mitad de los años 90, ha experimentado un desarrollo bastante tormentoso. Este desarrollo legal tiene dos caras como la cabeza de Jano: por un lado, está caracterizado por la humanización del Derecho de los conflictos armados no internacionales. Sin embargo, por otro lado, las reglas que gobiernan las conductas en hostilidades se han cristalizado, y su denominación como 'humanitaria' no deja de

Doctor en Derecho de la Universidad de Colonia (Alemania). Titular de la Cátedra de Derecho Penal Alemán, Derecho Internacional y Derecho Penal Internacional en la Universidad de Colonia; director del Instituto de Derecho Internacional de la Paz y la Seguridad de la Universidad de Colonia; miembro vitalicio de Clare Hall College de la Universidad de Cambridge (EEUU); miembro de la Academia de Ciencias y Artes de Renania del Norte-Westfalia; miembro de la Delegación de Alemania ante la Comisión Preparatoria de la Corte Penal Internacional y negociador sobre las Reglas de Procedimiento y Prueba (Nueva York). Köln, Alemania. Correo-e: claus.kress@uni-koeln.de. Fecha de recepción: 17 de marzo de 2017. Fecha de modificación: 26 de mayo de 2017. Fecha de aceptación: 31 de agosto de 2017. Para citar el artículo: KLAus KRESS. "La cabeza de Jano en la evolución del Ius in Bello Interno y la cuestión del Nuevo Ius Contra Bellum Internum”, en Revista Derecho Penal y Criminología, Vol. 38, n. ${ }^{\circ}$ 104, enero-junio de 2017, Bogotá, Universidad Externado de Colombia, pp. 179-202. DOI: https://doi.org/10.18601/ 01210483.v38n104.06 
ser eufemística. Vale la pena preguntar si el desarrollo de la ley de conflictos armados no internacionales debe complementarse con un Derecho contra el conflicto armado no internacional. La controversia presente sobre una política legal acerca del privilegio del combatiente para guerreros no estatales debe desenvolverse bajo esta nueva luz.

Palabras clave: Desarrollo histórico del Derecho del conflicto armado no internacional, Asimilación del Derecho del conflicto armado no internacional al Derecho del conflicto armado no internacional, Derecho consuetudinario del conflicto armado, Corte Penal Internacional para la antigua Yugoslavia y el desarrollo del Derecho del conflicto armado no internacional, Derecho internacional de los conflictos armados, Derecho internacional de los derechos humanos, Derecho que rige las conductas hostiles en el conflicto armado no internacional, Conflicto armado no internacional con dimensión transnacional, Asimetría normativa en el Derecho de conflictos armados no internacionales, Crimen de agresión, Privilegio de combatientes en el Derecho internacional de los conflictos armados, ius contra bellum internum.

\title{
THE HEAD OF JANO IN THE EVOLUTION OF INTERNAL IUS IN BELLO AND THE QUESTION OF THE NEW INTERNAL IUS CONTRA BELLUM INTERNUM
}

\begin{abstract}
Since 1945, most armed conflicts took place between states and rebels or between non-state armed groups within fragile or failed states. Currently, the dramas in Iraq, Syria and Ukraine show us that the civil war is one of the major evils of our time. Recognizing this fact, the right of non-international armed conflict, particularly since the first half of the $90 \mathrm{~s}$, has experienced a rather stormy development. This legal development has two faces like Janus head: one is characterized by the humanization of the law of non-international armed conflicts. However, on the other hand, the rules governing conduct in hostilities have crystallized, where his name as 'humanitarian' continues to be euphemistic. It is worth asking whether the development of the law of non-international armed conflict must be complemented by a right against non-international armed conflict. The present controversy over legal policy about the privilege to non-state Fighter warriors must operate in this new light.
\end{abstract}

Keywords: Historical development of the law of non-international armed conflict, Assimilation of the law of non-international armed conflict the right of non-international armed conflict, Customary law of armed conflict, International Criminal Court for the former Yugoslavia and the development of the law of non-international armed conflict, International law of armed conflict and international law of human rights, The law governing hostile behavior in non-international armed conflict, Non-international armed conflict with transnational dimension, Regulatory asymmetry on the right of non-international 
armed conflicts, The crime of aggression and privilege of combatants in international law of armed conflict, ius contra bellum internum.

\section{LA HUMANIZACIÓN ${ }^{1}$ DEL DERECHO DE LOS CONFLICTOS ARMADOS NO INTERNACIONALES}

La imagen devastadora de los soldados heridos, tirados sobre el campo de batalla cerca del pueblo de Solferino después de la batalla entre las fuerzas aliadas de Cerdeña y Francia por un lado, y las de Austria por el otro, despertó un impulso humanitario en el genovés Henry Dunant. En 1862, él mismo iluminó el faro con su obra $R e$ cuerdo de Solferino ${ }^{2}$. Dunant imploró de manera apasionada por el establecimiento de una sociedad voluntaria de auxilio dedicada al cuidado de los heridos en tiempos de guerra, así como porque esta institución innovadora se encontrara protegida a través de un acuerdo entre Estados. Para 1863, el que muy poco después se denominó Comité Internacional de la Cruz Roja (CICR), que celebró su 150 aniversario el año pasado, fue fundado y tan solo un año después fue adoptado el Convenio de Ginebra para para aliviar la suerte que corren los heridos y los enfermos de las fuerzas armadas en campaña ${ }^{3}$. Fuera de las tormentas intermitentes, un camino directo conduce de este primer convenio, al Derecho Internacional Humanitario de hoy ${ }^{4}$, cuya preocupación principal es -tal y como era en la segunda mitad del siglo XIV- aliviar el sufrimiento causado por el conflicto armado. Con todo, al principio, la única preocupación era la contención de la guerra (internacional); la regulación de la guerra civil que potencialmente no es menos desgarradora vendría más tarde. El Derecho internacional clásico no proveyó ninguna protección y, hasta el final de la Segunda Guerra Mundial, las guerras civiles quedaron fuera del alcance del Derecho internacional y con ello, eran domaine reservé del Estado afectado ${ }^{5}$.

1 El término, por supuesto, se toma prestado de THEOdOR MERON, "The Humanization of Humanitarian Law", American Journal of International Law, Vol. 94, 2000, p. 239. En este artículo, Meron (ibídem.) describe cómo el derecho de la guerra 'conducido en buena medida por los derechos humanos y los principios de humanidad (...) ha ido cambiando y adquiriendo cada vez más un rostro humano', y expone 'las incursiones hechas por el rol dominante de la reciprocidad; la acogida, la responsabilidad (accountability); y las innovaciones en la formación, formulación en interpretación de reglas'.

2 Jean-Henry Dunant, Un souvenir de Solférino, Cassel, London, 1947.

3 Auswärtiges Amt/Deutsches Rotes Kreuz/Bundesministerium der Verteidigung (eds), Dokumente zum Humanitären Völkerrecht, 2nd ed., Academia Verlag, Sankt Augustin, 2012, p. 13 ("Documents on International Humanitarian Law").

4 Para un relato del desarrollo histórico, ver MARY Ellen O'Connell, "Historical Development and Legal Basis", en Dieter Fleck (ed.), The Handbook of International Humanitarian Law, 3rd ed., Oxford University Press, Oxford, 2013,pp. 1 y ss.

5 En la práctica esto ciertamente no implica una completa ausencia de regulación. Por el contrario, las fuerzas armadas, a veces, recibían instrucciones internas. (Para mayores detalles, ver el brillante libro de Sandesh Sivakuraman, The Law of International Armed Conflict, Oxford University Press, 2012, pp. 9-29.) El código redactado por FrANCIS LIEBER, al que en 1863 Abraham Lincoln dio ca- 
En 1949, cuando las leyes de la guerra se sometieron a escrutinio como consecuencia de la catástrofe de la Segunda Guerra Mundial, el Comité Internacional de la Cruz Roja hizo un llamado para que la renovación del Derecho Internacional Humanitario se extendiera a la guerra civil. No solo Birmania consideró que esta propuesta iba demasiado lejos. Gran Bretaña, por ejemplo, expresó su preocupación recalcando la importancia de la soberanía nacional ${ }^{6}$. Al final, los Estados llegaron a un acuerdo sobre el texto del Art. 3 común, el 'mini-convenio', que regulaba la guerra civil al interior de cuatro mayores Nuevos Convenios de Ginebra (GC) en Derecho Internacional Humanitario ${ }^{7}$. La aceptación de este mini-convenio marcó el nacimiento de un ius bello interno (o en su expresión técnica legal -el Derecho internacional de conflictos armados no internacionales $)^{8}$. Cerca de tres décadas más tarde los Estados rechazaron otro intento de asimilar el Derecho Internacional Humanitario aplicable en los conflictos no internacionales, con aquel aplicable a los conflictos armados internacionales. Entre 1974 y 1947 la Conferencia Diplomática sobre reafirmación y Desarrollo del Derecho Internacional Humanitario aplicable en conflictos armados, que condujo a la adopción de dos Protocolos Adicionales (PA) a la CG, los Estados se encontraban divididos sobre la manera de clasificar las luchas de liberación anticolonial, lo que de acuerdo con una perspectiva clásica constituía una forma específica de guerra civil ${ }^{9}$. Los Estados que emergieron de estos conflictos, junto con los que los

rácter vinculante para las tropas de la Unión durante la Guerra Civil Americana, y que los Estados Confederados después aceptarían, es el más famoso ejemplo que subsecuentemente influenciaría el desarrollo del Derecho Internacional Humanitario en general. Más aún, era posible reconocer a los rebeldes que habían tenido éxito estableciendo estructuras cuasi estatales en ciertas partes afectadas por la guerra, como partes beligerantes en el conflicto (S. SivaKumaran ibídem., p.9). Como resultado de este reconocimiento, las leyes de la guerra se hicieron aplicables para las dos partes. Sin embargo, la cuestión de reconocimiento de rebeldes como una parte beligerante era a menudo un asunto controversial, y los estados eran reacios a aceptar una obligación de reconocimiento. Por ejemplo, en la Guerra Civil Española de 1930 el gobierno español se rehusó a reconocer su oponente como beligerante (S. SIVAKUMARAN, Ibid., p. 17; sobre el significado de la guerra civil española y su posterior desarrollo para derecho de los conflictos armados no internacionales, ver ANTONIO CASSESE. "The Spanish Civil War and the Development of Customary Law Concerning Internal Conflicts" en: Antonio CAssese (ed.), Current Problems of International Law. Essays on U.N. Law and on the Law of Armed Conflict, Dott. A. Giuffrè Editore, Milan, 1975, pp. 287 y ss.) La protección del individuo a través del Derecho internacional en guerras civiles siguió siendo precaria.

6 Ver S. SivaKumaran, arriba nota 5, p. 40 (con referencias en notas 77, 78).

7 Documents on International Humanitarian Law, arriba nota 3, p. 167; para un examen temprano de los Convenios de Ginebra, ver por ejemplo, Hersch LAUTERPACHT, "The Problem of the Revision of the Law of War", British Year Book of International Law, Vol. 29, 1952, pp. 360 ss. Aparte del Art. 3 común de los convenios de Ginebra, otras disposiciones se han limitado al conflicto armado internacional en el marco del significado del Art. 2 común de los Convenios de Ginebra.

8 Acerca de las negociaciones que llevaron a este acuerdo, ver S. SiVAKUMARAN, note 5, pp. 40 y ss.; Anthony Cullen, The Concept of Non-International Armed Conflict in International Humanitarian Law, Cambridge University Press, Cambridge, 2010, pp. 25 y ss; LINDSAY MoIR, The Law of Internal Armed Conflict, Cambridge University Press, 2002, pp. 23 y ss.

9 ANTONIO CASSESE, "Wars of National Liberation and humanitarian law", en CHRISTOPHE SwINARSKI (ed.), Études et essais sur le droit international humanitaire et sur les principes de la Croix-Rouge 
apoyaban, tuvieron éxito en "actualizar", así sea bajo estrictas condiciones, la lucha nacional de liberación como conflicto armado internacional ${ }^{10}$. Habiendo alcanzado esta meta, la sed de reforma de los Estados no alineados fue esencialmente satisfecha. Especialmente, los nuevos Estados independientes, cuyos gobiernos frecuentemente se confrontaron tensiones internas, no se tomaron mucho tiempo en reconocer el atractivo en la protección de la soberanía internacional a través del Derecho Internacional. India lo expresó de modo franco en esta conferencia: tras saldar la cuestión de las luchas de liberación nacional, no había necesidad de extender la regulación del Derecho internacional de los conflictos armados no internacionales ${ }^{11}$. Más allá de estas declaraciones, los Protocolos Adicionales II de los conflictos armados no internacionales fueron adoptados con el tiempo. Su contenido, sin embargo, quedó con un alcance limitado.

No obstante, la expansión del Derecho de los conflictos armados no internacionales llegó con una gran fuerza. Ella no tomó la forma ceremonial de la conclusión diplomática de un tratado pero sí tuvo lugar en un camino sin intromisiones similar al del Derecho internacional no escrito. Con miras a desplegar cierta efectividad confiable en la práctica, este Derecho internacional consuetudinario debe ser identificado autoritativamente - preferiblemente por una corte internacional. La Corte Penal Internacional para la antigua Yugoslavia (TPIY) que, a la luz de las atrocidades cometidas en los Balcanes, fue establecida por el Consejo de Seguridad de Naciones Unidas en 1993, se erigiría a sí misma como la institución clave que progresivamente determinaría la existencia de Derecho internacional consuetudinario en asuntos de Derecho de los conflictos armados no internacionales. La elección del Profesor Antonio Cassese como juez del TPIY se mostraría como determinante en este proceso. Cassese no solo tenía en general una mentalidad progresista, sino que era partidario de desarrollar un Derecho internacional aplicable en las guerras civiles ${ }^{12}$. El momento de Cassese llegó cuando el primer acusado, Duško Tadić, desafió la jurisdicción de la TPIY para determinar su responsabilidad criminal conforme con el Derecho internacional, en el conflicto interno en Yugoslavia, por falta de una ley suficientemente desarrollada para conflictos armados no internacionales. En una revolucionaria decisión del 2 octubre de 1995, el profesor de Derecho internacional, en toga de juez, realizó un examen comprensivo de la práctica internacional relacionada con la guerra civil desde 1936, y con la ingenuidad de un verdadero maestro identificó una tendencia omnipresente

en l'honneur de Jean Pictet, ICRC/Martinus Nijhoff Publishers, Geneva/The Hague, 1984, pp. 313 y ss.

10 Art. 1(4) y Art. 96 AP I; Documents on International Humanitarian Law, arriba nota 3, pp. 503, 509, and 575; sobre la posición de los Estados no alineados ver LUIGI CONDORELLI, "Pays Afro-Asiatiques", en Antonio Cassese (ed.), The New Humanitarian Law of Armed Conflict, Editoriale Scientifica S.r.l., Naples, 1979, pp. 386 y ss., y en particular, pp. 392 y ss.

11 S. SivAKumaran, arriba nota 5, p. 50 (con referencia en la nota 150).

12 Ver sobre todo su contribución temprana. A. CASSESE, arriba nota 5, pp. 287 y ss, y en particular, pp. 315 y ss. 
por la que las partes en guerras civiles aceptan de manera creciente reglas que son similares a aquellas que rigen las guerras interestatales.

De acuerdo con Cassese y sus colegas jueces, la distinción entre guerras sofisticadas (que rigen guerras interestatales) y las rudimentarias reglas que regulan el conflicto armado no internacional "tenía una orientación sobre la soberanía" 13 y "si el Derecho internacional... gradualmente debe girar hacia la protección de los seres humanos"14, la llamada "dicotomía debe perder gradualmente su peso" 15 . Claramente, la decisión Tadić fue animada por el caso por el espíritu de Henry Dunant y su mensaje central prevaleció rápidamente ${ }^{16}$. En el 2005, el CICR unió su voz al destacable desarrollo que había tenido el Derecho internacional consuetudinario de los conflictos armados ${ }^{17}$. El guardián del Derecho Internacional Humanitario confirmó la asimilación del Derecho internacional consuetudinario de los conflictos armados no internacionales al Derecho de los conflictos armados internacionales.

La "dinámica Tadić" (esto es, la asimilación del Derecho de los conflictos armados no internacionales a aquella que rige para los conflictos internacionales, con el objeto de reforzar la anterior protección humanitaria) estaba alineada con otro desarrollo fundamental en el Derecho internacional: el desarrollo de un estándar mínimo de Derecho consuetudinario internacional para la protección de los derechos humanos, en particular, después de la adopción en 1966 de los dos Pactos Internacionales para la protección de los derechos humanos ${ }^{18}$. No sorprendía que Cassese se apoyara en la cristalización de Derecho internacional de los derechos humanos, pues nada podía dejar tan claro que el Derecho internacional se abría de manera más directa a las preocupaciones del individuo. Más aún, después de cierta incertidumbre se reconoció cada vez más que se podía contar el Derecho internacional de los derechos humanos para enriquecer y fortalecer el Derecho Internacional Humanitario de los conflictos armados no internacionale ${ }^{19}$. El segundo reporte proferido por la comisión

13 CIJY, The Prosecutor v. Duško Tadić, Caso No. IT-94-1-AR72, 2 octubre 1995, para. 96.

14 Ibídem., para. 97.

15 Ibídem.

16 Claus Kress, "War Crimes Committed in Non-International Armed Conflict and the Emerging System of International Criminal Justice”, Israel Yearbook on Human Rights, Vol. 30, 2000, pp. 104-109.

17 Jean-Marie Henckaerts and Louise Doswald-Beck (eds), Customary International Humanitarian Law, Vol.I: Rules. Vol. II: Practice, Cambridge University Press, Cambridge, 2005; para una posición más reservada sobre el estatus del derecho consuetudinario que rige el derecho del conflicto armado no internacional ver JEAN D’ ASPRÉMONT and JÉRÔME DE HEMPTINNE, Droit international humanitaire, Editions A. Pedone, Paris, 2012, pp. 25 y ss.

18 Ver por ejemplo, Thomas Buergenthal, "Human Rights", en RüDiger Wolfrum (ed.), The Max Planck Encyclopedia of Public International Law. Vol. IV, Oxford University Press, 2012, pp. 1023 y ss.

19 Para un análisis del asunto ver RoBert Kolb, "Human Rights and Humanitarian Law", en R. WoLFRUM, Ibídem., pp. 1.040 y ss. 
independiente Israelí Turkel en 2012 ejemplificó hasta qué punto esto podía ocurrir. En completo acuerdo con la "dinámica Tadić”, la comisión llegó a la conclusión de que había surgido un deber de Derecho internacional consuetudinario, según el cual el Estado territorial y aquel de nacionalidad activa, debían investigar aquellas acusaciones creíbles de crímenes de guerra cometidos en conflictos armados no internacionales.

Más aún, la Comisión sostuvo que el Derecho internacional de los derechos humanos podría proveer una orientación importante al establecer en este deber de investigar contenidos significativos ${ }^{20}$. Para concluir, la humanización del Derecho internacional de los conflictos armados no internacionales ha alcanzado un avanzado estado en la actualidad $^{21}$.

\section{LA TENSIÓN ENTRE EL DERECHO DE CONFLICTOS ARMADOS NO INTERNACIONALES Y EL DERECHO DE LOS DERECHOS HUMANOS}

La humanización es, sin embargo, solo una cara de la moneda. Si se toma como un todo, la evolución legal bajo consideración ha devenido profundamente ambivalente. La razón es que esta evolución se ha difundido a los ámbitos de conducción de hostilidades $^{22}$ que forma parte importante de las leyes de la guerra.

El Derecho que rige la conducción de hostilidades en el conflicto armado internacional

Si de cierta manera se examina más de cerca el Derecho sobre conducción de hostilidades en conflictos armados internacionales ${ }^{23}$, los componentes clave que se han codificado en el PAI, el término "Derecho Internacional Humanitario" revela de cierta manera un aspecto eufemístico. El Derecho acerca de las conducción de hostilidades en conflictos armados internacionales permite matar a los combatientes enemigos ${ }^{24}$ durante el conflicto, indiferentemente de que el combatiente a quien se apunta se disponga (o no) a lanzar un ataque. Esta permisión no responde a un peligro inmediato

20 La Comisión pública para examinar el Incidente Marítimo de mayo 31 de 2010, Segundo Reporte: Mecanismos de Israel para examinar las quejas y los reclamos de violación del derecho del conflicto armado de acuerdo con el Derecho internacional, febrero 2013, p. 93 y en particular, pp. 114 y ss.

21 Para un recuento comprensivo del Derecho sobre la materia, ver S. SIVAKUMARAN, arriba nota 5, pp. 255-335; ver también pp. 430 y ss.

22 Para un recuento comprensivo del Derecho del conflicto armado no internacional que rige la conducción de hostilidades, ver S. SIVAKUMARAN, arriba nota 5, pp. 336-429.

23 Para un tratamiento monográfico, ver YorAm DinsteIn, The Conduct of Hostilities under the Law of International Armed Conflict, 2. ed., Cambridge University Press, Cambridge, 2010, passim; para un recuento más conciso, ver Stefan Oeter, "Methods and Means of Combat", en D. FLECK, arriba nota 4 , pp. 115 y ss.

24 Para el concepto jurídico de combatiente ver, por ejemplo "Combatants and Non-Combatants", en D. FLECK, arriba nota 4, pp. 85 y ss. 
causado por un ser humano individual, pero refleja la pertenencia a una colectividad/ grupo que está preparada para pelear.

A la luz del ideal de los derechos humanos, resulta mucho más difícil de digerir el que el Derecho sobre la conducción de hostilidades no ofrezca completa protección a los civiles. De acuerdo con el principio legal más básico, tal y como se encuentra codificado en la primera oración del Art. 51 (2) PA I, los civiles no deben ser objeto del ataque. Sin embargo, el ataque sobre un objetivo militar sigue siendo permisible si bajo ciertas circunstancias y después de tomar todas las debidas medidas de precaución, persiste el riesgo de daños colaterales sobre la población civil. El principio consuetudinario de ius in bello, proporcionalidad, tal y como está codificado en el Art. 51 (5) PA I, solo prohíbe un ataque, "cuando sea de prever que causarán incidentalmente muertos y heridos entre la población civil (...) que serían excesivos en relación con la ventaja militar concreta y directa prevista ${ }^{25}$.

Además, la ley que está relacionada con los prisioneros de guerra constituye una institución jurídica de doble filo. Por humanitaria que pueda ser la prohibición absoluta de tortura de prisioneros de guerra, es obvio que el marco legal autoriza una detención preventiva extraordinariamente robusta de miembros individuales de una colectividad -las fuerzas armadas enemigas. Este tipo de detención no se basa en decisiones judiciales y se limita únicamente al fin del conflicto. A pesar de los cantos de sirena del término Derecho Internacional Humanitario, este cuerpo de leyes reconoce las necesidades de la guerra en una extensión considerable. Como se ha observado una y otra vez, la ley del conflicto armado internacional no puede dirigirse contra el acto de guerra como tal. Se acepta la incómoda verdad de que la victoria en una guerra de autodefensa perfectamente justificada a menudo no puede ser asegurada si las hostilidades tienen que ser conducidas de acuerdo con el ideal de los derechos humanos. Es este estado de mayor emergencia el que exige que el Derecho internacional de conflictos armados abrace un paradigma permisivo de conducción de hostilidades que, de modo severo, recorta el alcance de los derechos a la vida y a la libertad, internacionalmente garantizados.

\section{EL DERECHO QUE REGULA LA CONDUCCIÓN DE HOSTILIDADES EN EL CONFLICTO ARMADO NO INTERNACIONAL}

La cuestión acerca de si el Derecho de los conflictos armados no internacionales incluye un marco jurídico para la conducción de hostilidades ha permanecido sin

25 Stefan Oeter, "Methods and Means of Combat", en D. Fleck, arriba nota 4, pp. 196 y ss; para un fascinante análisis reciente de los daños "colatares civiles" en el Derecho internacional y en ética ver Reinhard Merkel, “Die ‘kollaterale’ Tötung von Zivilisten im Krieg. Rechtsethische Grundlagen und Grenzen einer prekären Erlaubnis des humanitären Völkerrechts“, Juristen Zeitung, Vol. 67, 2012, pp. 1137 y ss. (disponible en alemán). 
respuesta por largo tiempo ${ }^{26}$. Posiblemente, la ausencia de un estándar vinculante de derechos humanos hasta la segunda mitad del siglo xx, sea una razón para esta incertidumbre. En la medida que los Estados no están sujetos a obligaciones internacionales de derechos humanos, sus gobiernos no sienten una urgente necesidad de contemplar el Derecho internacional cerca de la conducción de hostilidades en conflictos armados no internacionales. Desde la perspectiva de un gobierno que afronta tensiones internas con potencial escalamiento, debe ser una opción atractiva el estar en posición de rechazar el escrutinio internacional sobre la manera como debe ser manejado el conflicto con los opositores, argumentando que esto queda dentro del domaine réservée del Estado involucrado. Después de la Segunda Guerra Mundial, como es bien conocido, un cálculo similar ha llevado repetidamente a los Estados a negar, contrario a los hechos, la existencia de un conflicto armado no internacional en el territorio respectivo. La admisión de un conflicto armado no internacional no solo mostraría un desafío a su autoridad sino que implicaría el reconocimiento de aplicabilidad de los estándares internacionales formulados en el Art. 3 Común de los Convenios de Ginebra.

Tal y como lo ha establecido David Kretzmer en un iluminador análisis, la imagen cambió radicalmente una vez se puso un estándar internacional vinculante de derechos humanos 27 .

Los gobiernos que se confrontaron directamente con grupos de rebeldes bien organizados tuvieron que caer en la cuenta de que bajo el pleno efecto restrictivo de los derechos a la vida y la libertad, la victoria en la guerra civil era tan incierta como en una guerra de autodefensa contra las fuerzas armadas de un Estado agresor. Esta verdad se aplica a los Estados externos que asisten a los gobiernos que se encuentran amenazados. Alemania, por ejemplo, experimentó este dilema mucho antes de que su establecimiento lo esperara. La memoria de un lento y doloroso proceso alemán de aprendizaje de haber hecho parte de un conflicto no internacional aún está vi$\mathrm{va}^{28}$. Mientras los políticos alemanes silenciosamente seguían tratando de desechar el dulce sueño de una misión "suave" de asistencia en seguridad en Afganistán, la orden del coronel Klein de atacar en el Kunduz forzó a la judicatura alemana a aceptar una realidad menos feliz en el campo ${ }^{29}$. Dado que el ataque en el valle del río

26 S. SivAKUMARAN, arriba nota 5, p. 336; A. CASSESE, arriba nota 8, p. 294 y ss, ha buscado demostrar, sin embargo, que - a pesar del no reconocimiento de beligerancia- la conducta de hostilidades de las dos partes en la Guerra Civil Española en los años treinta, era escrutada sobre la base de principios legales parecidos a aquellos que rigen las leyes de la Guerra.

27 DAVID KRETZMER. "Rethinking the Application of IHL in Non-International Armed Conflicts", Israel Law Review, Vol. 42, 2009, pp. 8 y ss.

28 "What is this thing called war?", The Economist, 22 April 2010; http://www.economist.com/ node/15954464/print?story_id=15954464. Última visita: 13 de marzo de 2014.

29 En horas tempranas del 4 de septiembre de 2009, el Coronel Klein, que hacía parte de un contingente de la Fuerza Internacional de Asistencia Internacional en Seguridad (German International Security 
Kunduz se conformaba con los criterios de delito de asesinato según la sección 211 del Código Penal Alemán, y como la conducta no pudo justificarse de acuerdo con el paradigma de ejecución legal, el fiscal de la República Federal, para defender al coronel se soportó en el Derecho internacional consuetudinario sobre el manejo de hostilidades en conflictos armados no internacionales ${ }^{30}$. De cara a las casualidades civiles, resulta difícil ver aquí el ímpetu de un Henry Dunant trabajando.

Y con todo, sobre la base del vigente Derecho internacional de conflictos armados no internacionales, el fiscal estaba esencialmente en lo correcto. Hoy en día, posiblemente, la mayoría de los Estados comparten el punto de vista del Fiscal de la República Federal y reconocen que en una época de universal protección a los derechos humanos por parte del Derecho internacional, se necesita también un régimen legal especial relacionado con la conducción de hostilidades en conflictos armados no internacionales. La Corte Internacional de Justicia (CIJ) aceptó esto en su opinión consultiva sobre armas nucleares, en la medida en que determinó que el Derecho sobre la conducción de hostilidades limita el efecto del derecho humano a la vida en todos los conflictos armados y, por tanto, también en los conflictos armados no internacionales ${ }^{31}$. En el año 2009, el CICR siguió la misma línea. En su fundante Guía Interpretativa sobre la noción de participación en hostilidades bajo el Derecho Internacional Humanitario, precisó los componentes claves del Derecho que rige la conducción de hostilidades en conflictos armados no internacionales, y con ello promocionó la existencia de este régimen jurídico especial y su similitud con el correspondiente cuerpo jurídico aplicable a los conflictos armados internacionales ${ }^{32}$. Finalmente, en septiembre del año pasado, Christof Heyns, en su función como relator especial de Naciones Unidas para las ejecuciones extrajudiciales, sumarias o arbitrarias, reconoció la existencia de un marco jurídico especial internacional que rige la conducción de hostilidades en conflictos armados no internacionales, que

Assistance Force) (ISAF), actuando bajo el mandato del Consejo de Seguridad de Naciones Unidas, ordenó un ataque aéreo sobre dos camiones de combustible que habían sido secuestrados por fuerzas talibanes y que se habían atascado en un banco de arena en la mitad del río Kunduz. De acuerdo con la narración de los hechos por parte del Fiscal General Alemán, ante el Tribunal Supremo, el Coronel intentó destruir los camiones (respecto de los cuales tenía razones para creer que constituían una amenaza) y sabía que el ataque también golpearía un número de comandantes talibanes presentes en el lugar, sin esperar que cayeran víctimas civiles; Der Generalbundesanwalt beim Bundesgerichtshof, Ermittlungsverfahren gegen Oberst Klein und Hauptfeldwebel Wilhelm wegen des Verdachts einer Straftat nach dem VStGB und anderer Delikte, 3 BJs 6/10-4, Offene Version des Vermerks vom 16.4.2010 zur Verfügung der Einstellung nach $§ 170$ Abs. 2 Satz 1 StPO, p. 13 y ss.

30 Der Generalbundesanwalt beim Bundesgerichtshof, Ibid., p. 41 y ss.

31 ICJ,Legality of the Threat or Use of Nuclear Weapons (Advisory Opinion), I.C.J. Reports 1996, 226, 240 (para. 25).

32 CICR, Guía Interpretativa sobre la noción de participación en hostilidades bajo el Derecho Internacional Humanitario Ginebra, Mayo 2009. 
limita el efecto pleno de los derechos humanos ${ }^{33}$. El reporte de Heyn, que de modo circunspecto da cuenta del debate en Derecho internacional sobre el uso de drones en situaciones de conflicto armado y más allá, es particularmente significativo a la luz del mandato de derechos humanos de este Relator Especial de Naciones Unidas.

\section{LA POSICIÓN LEGAL DEL GOBIERNO DE LOS ESTADOS UNIDOS DE AMÉRICA}

Aquí es donde la Administración Obama comienza con la parte ius in bello ${ }^{34}$ de justificación de su política de homicidios selectivos en su lucha contra "Al Qaeda y sus fuerzas asociadas", y con ello, viene a la luz, con particular claridad, el lado no tan humanitario del "paradigma jurídico del conflicto armado no internacional". Nadie tuvo que exhortar a los Estados Unidos de América para que admitieran estar envueltos en un conflicto armado no internacional. Por el contrario, el gobierno de Estados Unidos, soportado por la Corte Suprema Justicia ${ }^{35}$, ha estado más que dispuesto a aceptar su participación en este conflicto armado. De hecho, este punto de vista se ha convertido en la piedra angular de la posición jurídica que los Estados han desarrollado en el tiempo para justificar el uso de drones contra (presuntos) objetivos terroristas en Afganistán, Paquistán y otros países ${ }^{36}$. Es por ello que, también en el

33 U.N. Doc. A/68/382, 13.9.2013, para. 52 y ss. Debe anotarse, quizá, que el reporte de Heyns (lo que es cierto para la academia en Derecho internacional) no clarifica del todo la posición legal de los soldados en conflictos armados no internacionales respecto de un asunto que es importante tanto en la teoría como en la práctica. Mientras que es difícil negar que la práctica estatal apoye la posición de que el Derecho de los conflictos armados no internacionales faculte al Estado para ir más allá de los límites de los derechos humanos al combatir a su adversario, resulta poco claro si el soldado individual puede disfrutar del privilegio de apuntar bajo el Derecho del conflicto armado no internacional o si esta facultad solo puede emanar de una obra de legislación doméstica del Estado en cuestión que, por así decirlo, activa un paradigma de Derecho no internacional para el conflicto armado; incluso, Sandesh Sivakumaran evita una decisión con respecto a este asunto en su monumental obra (ver S. SIVAKUMARAN, arriba nota 5, p. 151).

34 Acerca "ius ad bellum-part", ver Claus KRESS, "Major Post-Westphalian Shifts and Some Important Neo-Westphalian Hesitations in the State Practice on the International Law on the Use of Force", Journal on the Use of Force and International Law, Vol. 1, 2014, p. 37 y ss.

35 Hamdan v Rumsfeld. Secretary of Defense et al., (2006) 126 S Ct 2746, 2757.

36 La posición actual de los Estados Unidos puede encontrarse en una serie de pronunciamientos públicos de funcionarios del gobierno, que comienzan con los Observaciones de marzo 25 de 2010 por el entonces asesor jurídico Harold Honju Koh, en la Reunión Anual de la Sociedad Americana de Derecho Internacional. (http://www.state.gov/s/1/releases/remarks/139119.htm) y que culmina con el discurso del 23 de mayo de 2013, dado por el Presidente Obama en Universidad de Defensa Nacional (National Defense University) (Washington, D. C.) (http://www.whitehouse.gov/the-pressoffice/2013/05/23/remarks-president-national-defense-university); para otros importantes pronunciamientos, ver John O. BREnNAn, discurso del 30 de abril de 2012, en el Woodrow Wilson International Center for Scholars: “The Ethics and Efficacy of the President's Counterterrorism Strategy" (http:// www.wilsoncenter.org/event/the-efficacy-and-ethics-us-counterterrorism-strategy); Stephen Preston, General Counsel, Central Intelligency Agency, discurso del 10 de abril del 2012 en Harvard Law 
contexto de la política de Estados Unidos sobre homicidios selectivos, el Derecho internacional de los conflictos armados no internacionales no funciona principalmente como una limitación a la acción del Estado sino como una autorización jurídica para una acción robusta debido a una presunta necesidad militar. Junto con la "dinámica Tadic" que ha prevalecido por largo tiempo, reclama su propio derecho una "dinámica Al Qaeda" detrás de los desarrollos del Derecho de los conflictos armados no internacionales (p.ej una asimilación del Derecho de los conflictos armados no internacionales con el Derecho del conflicto armado internacional, que tiene como objeto facultar a los Estados para iniciar acciones coercitivas más allá de las limitaciones de paz del Derecho Internacional Humanitario).

El paradigma jurídico del conflicto armado no internacional, tal y como ha sido aplicado por los Estados Unidos en la lucha contra "Al Qaeda y sus fuerzas asociadas", tiene un mayor alcance que las demandas jurídicas que otros Estados han formulado para conflictos anteriores en los que estaban involucrados. Los cuatro quizá más importantes componentes de la posición de Estados Unidos son los siguientes. En primer lugar, este Estado sostiene que un conflicto armado no internacional puede asumir una dimensión transnacional ${ }^{37}$. En segundo lugar, los Estados Unidos niegan la existencia de cualquier limitación geográfica en el ámbito de aplicación de los poderes de señalamiento de objetivos (targeting powers) bajo las reglas que rigen la conducción de hostilidades en un conflicto armado no internacional (transnacional) ${ }^{38}$.

School: "CIA and the Rule of Law" (http://www.cfr.org/rule-of-law/cia-general-counsel-stephenprestons-remarks-rule-of-law-april-2012/p27912); ERIC HOLDER. Attorney General, Observaciones del 5 de marzo de 2012 en el Northwestern University Law School (http://lawfareblog.com/2012/03/ text-of-the-attorney-generals-national-security-speech/\#mare-6236); JEH JoHnSON, General Counsel, us Department of Defense, Dean's Lecture del 22 de febrero de 2012 en Yale Law School: "National Security Law, Lawyers and Lawyering in the Obama Administration" (http://lawfareblog.com/2012/02/ jeh-johnson-speech-at-yale-law-school); JoHn O. Brennan, Assistant to the President for Homeland Security and Counterterrorism, Observaciones del 16 de septiembre de 2011 en Harvard Law School Program on Law and Security: "Strengthening Our Security by Adhering to Our Values and Laws" (http://www.whitehouse.gov/the-press-office/2011/09/16/remarks-john-o-brennan-strengtheningour-security-adhering-our-values-an) (últimas visitas en estos sitios: 18 de junio de 2014).

37 Para esta parte del argumento de Estados Unidos, ver, de forma concurrente S. SIVAKUMARAN, arriba nota 5, pp. 228 y ss.; NoAm Lubell. Extraterritorial Use of Force Against Non-State Actors, Oxford University Press, Oxford, 2010, p. 99 y ss.; Claus KRESS, "Some Reflections on the International Legal Framework Governing Transnational Armed Conflict", Journal of Conflict \& Security Law, Vol. 15, 2010, p. 255 y ss.; Andreas Paulus/Mindia Vashakmadze. "Asymmetrical War and the Notion of Armed Conflict: A Tentative Conceptualization", International Review of the Red Cross, Vol. 91, 2009, p. 112 y ss.

38 Acerca de esta parte del argumento de Estados Unidos ver, por un lado, NoAm LUbELL AND NATHAN DEREJKO. "A Global Battlefied. Drones and the Geographical Scope of Armed Conflict", Journal of International Criminal Justice, Vol. 11, 2013, p. 65 y ss.; y por el otro lado, MARY ELLEN O'CONNELL. “Combatants and Combat Zone”, University of Richmond Law Review, Vol. 43, 2008-2009, p. 863; para la posición del CICR ver la entrevista del Presidente Peter Maurer del 10 de mayo de 2013 (http:// www.icrc.org/eng/resources/documents/interview/2013/05-10-drone-weapons-ihl.htm; (última visita: 13 de julio de 2014); para una presentación y discusión de los argumentos ver CLAUS KrESS. "Re- 
En tercer lugar, los Estados Unidos suman la violencia que emana de una red internacional de grupos terroristas, unida por una ideología común al momento de determinar la existencia de un conflicto armado transnacional no internacional, sin aceptar, como requisito jurídico, la necesidad de $u n a$ orden central cuasi militar ${ }^{39}$. En cuarto lugar, los Estados Unidos, sin usar exactamente la misma terminología, como se ha sugerido en la Guía Interpretativa sobre la noción de participación directa en hostilidades bajo el Derecho Internacional Humanitario ${ }^{40}$, asumen la perspectiva de que los actores armados no estatales con una función continua de combate pueden ser objetivo, más allá de las limitaciones temporales que se aplican (bajo los así llamados actos específicos de aproximación ${ }^{41}$ ) a los civiles que participan directamente en las hostilidades ${ }^{42}$. Si se toman conjuntamente, estos cuatro componentes ${ }^{43}$ se acumulan en una construcción jurídica que de manera significativa expande la aplicabilidad del Derecho permisivo del conflicto armado no internacional. No se puede negar que en los años cincuenta Jean Pictet, el gran comentador de la CG argumentó con firmeza para conceder al Derecho Internacional Humanitario un ámbito de aplicación tan amplio como fuera posible ${ }^{44}$. Para ese tiempo, sin embargo, Pictet argumentó desde el punto de vista humanitariamente limitado de la “dinámica Tadić”. Con la conciencia de la "dinámica Al Qaeda", se puede asumir que Pictet reconsideraría sus anteriores

view of Books", en British Yearbook of International Law, Vol. 83, 2012, p. 152 y ss.; ver también la declaración del Fiscal General Alemán en la Fiscalía ante el Tribunal Supremo Drohneneinsatz vom 4. Oktober 2010 in Mir Ali/Pakistan, Verfügung des Generalbundesanwalts vom 20. Juni 2013 - 3 Bjs 7/12 - 4, sub D. II. 1. (una traducción inglesa de la decisión puede encontrarse próximamente en International Law Reports, Vol. 157).

39 Para una fuerte crítica del argumento, ver Christof Heyns. U.N. Doc. A/68/382, 13.9.2013, paras. 59-63; N. Lubell, arriba nota 37, pp. 118 y ss.

40 CICR, arriba note 32 , pp. 71 y ss.

41 CICR, Ibid., p. 44

42 Para un análisis crítico de esta parte de la Guía Interpretativa, ver KenNETH WATKIN, "Opportunity Lost: Organized Armed Groups and the ICRC 'Direct Participation in Hostilities' Interpretive Guidance”, New York University Journal of International Law and Politics, Vol. 42, 2010, pp. 641 y ss.; para una respuesta ver, NILS MELZER, "Keeping the Balance Between Military Necessity and Humanity: A Response to Four Critiques of the ICRC's Interpretive Guidance on the Notion of Direct Participation in Hostilities", Ibid., pp. 837 y ss.

43 Adicionalmente, los Estados Unidos no han aceptado la sugerencia de interpretación hecha por CICR arriba nota 32, pp. 77 y ss., de que los principios de necesidad y humanidad exigen que el combatiente capture a un soldado enemigo en lugar de matarlo cuando esta captura es factible sin ponerlo o poner a los civiles en riesgo adicional. Para una fuerte crítica de esta sugerencia, ver W. HAYS PARKS. "Part IX of the ICRC 'Direct Participation Study': No Mandate, No Expertise, and Legally Incorrect", New York University Journal of International Law and Politics, Vol. 42, 2010, pp. 769 y ss.; para una seca respuesta, ver N. MELzER, arriba nota 42, pp. 892 y ss.; para una útil discusión anterior, ver Robin Geiss. “Military Necessity: A Fundamental ‘Principle’ Fallen Into Oblivion”, en HÉLÈnE Ruiz FABri, RÜDiger Wolfrum, JANA Gogolin (eds.), Select Proceedings of the European Society of International Law, Hart Publishing, Oxford and Portland, 2008, pp. 554 y ss.

44 Jean S. Pictet. The Geneva Conventions of 12 August 1949. Commentary: IV Geneva Convention Relative to the Protection of Civilian Persons in Time of War, Geneva, 1958, p. 36. 
apelaciones y encontraría un mérito en la reciente insistencia del Relator especial de Naciones Unidas Heyn, de que la "Paz debe ser una norma" 45.

\section{¿UN NUEVO DERECHO INTERNACIONAL CONTRA EL CONFLICTO ARMADO NO INTERNACIONAL COMPLEMENTADO POR UNA LEY SIMÉTRICA DE CONFLICTO ARMADO NO INTERNACIONAL?}

Como sea el estado del desarrollo jurídico en detalle, no se puede seguir negando que el Derecho no internacional de los conflictos armados ha pasado por un proceso notable de asimilación con el Derecho que rige el conflicto armado internacional, incluso respecto al área particularmente sensible de la conducción de hostilidades. Esto ha reanimado el debate académico acerca de la cuestión de si hay un retraso en conceder a los actores armados no estatales el privilegio de combatientes equivalente al de los soldados enfrentados en el conflicto armado. Como resultado de este cambio en el Derecho vigente, estos rebeldes que luchan atendiendo el Derecho de los conflictos armados no internacionales no solo se liberarán (como es el caso en la actualidad) de responsabilidad penal, sino que (contrario al Derecho existente) no serán castigados bajo el Derecho del respectivo Estado (dígase por rebelión o asesinato), dada su participación en el conflicto armado. En una de sus últimas publicaciones, Antonio Cassese ha implorado por una reforma en el caso de conflictos armados no internacionales de alta intensidad, manifestando que las fuerzas armadas de un participante no estable están suficientemente preparadas para distinguirse a sí mismas de la población civil ${ }^{46}$.

El argumento principal a favor de la introducción de privilegio de combatiente para los alzados en armas no estatales, en un conflicto armado no internacional, es de naturaleza humanitaria. El argumento es el siguiente: ¿qué incentivo puede haber para las fuerzas armadas no estatales en obedecer las reglas internacionales humanitarias si aquellas están condenadas a enfrentar un castigo al final del conflicto armado, a menos que prevalezcan militarmente ${ }^{47}$. Tan razonable como pueda resultar esta

45 U.N. Doc. A/68/382, 13 September 2013, para. 16.

46 Antonio Cassese. "Should Rebels be Treated as Criminals? Some Modest Proposals for Rendering Internal Armed Conflicts Less Inhumane", en Antonio CAssese (ed.), Realizing Utopia. The Future of International Law, Oxford University Press, Oxford, 2012, pp. 523 y ss.; para una súplica casi apasionada en favor de la misma propuesta de reforma, ver EMILY CRAWFORD, The Treatment of Combatants and Insurgents under the Law of Armed Conflict, Oxford University Press, Oxford, 2010, p. 168; más cauto, pero apuntando en la misma dirección S. SiVAKUMARAN, arriba nota 5, p. 520, sugiere aplicar el Art. 6 (5) 5 PA II de una manera que va a operar como un equivalente funcional de un privilegio de combatiente; para una propuesta temprana aún más modesta en la misma dirección, ver Art. 10 (5) de Draft Protocol II, Draft Additional Protocols to the Geneva Conventions de agosto 12, 1949, como se redactó por el CICR en 1973.

47 Antonio Cassese. "Should Rebels be Treated as Criminals? Some Modest Proposals for Rendering Internal Armed Conflicts Less Inhumane”, en A. CASSESE, Ibídem. 
pregunta, los Estados no tienen ninguna duda sobre cómo responder apropiadamente. Ellos pueden apreciar bien la perspectiva de un mejor incentivo para fuerzas armadas no estales para acogerse al Derecho Internacional Humanitario. Con todo, insisten en la necesidad de proveer un incentivo efectivo para que una oposición política no se alce en armas y se desencadene un conflicto armado no internacional ${ }^{48}$. A la luz del sufrimiento que se asocia con un conflicto de este tipo, esta respuesta tiene el mayor peso humanitario. ¿Se puede superar este callejón sin salida?

\section{ESBOZO DE DOS POSIBLES INNOVACIONES LEGALES}

Vale la pena mirar el Derecho que rige las relaciones interestatales para encontrar una posible salida. Aquí hay una tensión similar entre el objeto de prevenir, en primer lugar, el uso ilegal de la fuerza, y la necesidad de proporcionar protección humanitaria si este primer objetivo no es alcanzado. No obstante, el Derecho internacional vigente sobre paz y seguridad trata de moderar esta tensión de la siguiente manera. No solo prohíbe el uso de la fuerza (que queda para un número limitado de excepciones ${ }^{49}$ ), sino que también criminaliza, a pesar del crimen de agresión, la participación individual en casos serios del uso ilegal de la fuerza. Con todo, la amenaza de un castigo internacional se encuentra limitada para aquellos que se encuentran en "una posición de ejercer efectivamente el control sobre o de dirigir la acción política militar de un Estado" 50 . Al interior del sistema jurídico, el "privilegio" de combatiente mantiene también su potencial para que las fuerzas armadas del Estado agresor puedan conformarse con el Derecho Internacional Humanitario. En lo que tiene que ver con las relaciones entre Estados, el Derecho internacional apunta al liderazgo estatal para prevenir una guerra, y si esta prevención falla, les proporciona a las fuerzas armadas un incentivo para librar la guerra del modo más humanitario posible ${ }^{51}$.

48 Este punto es apreciado de forma completa por NILS MELZER. "Bolstering the Protection of Civilians in Armed Conflict", en A. CASSESE, arriba nota 46, p. 516.

49 El derecho a la autodefensa, tal y como está reconocido en el Art. 51 de la Carta de Naciones Unidas, y el uso de la fuerza, como se autoriza por el Consejo de Seguridad bajo el capítulo viI de la Carta de Naciones Unidas bajo el capítulo vil de la Carta de Naciones Unidas son dos excepciones universalmente aceptadas.

50 El juicio del primero de octubre de 1946 proferido por Tribunal Militar Internacional de Nuremberg sentó el precedente de criminalizar bajo el Derecho internacional el librar la guerra de agresión (para los pasajes claves del juicio y el posterior debate internacional sobre la materia, ver STEFAN BARRIGA/ Claus Kress (eds.), The Travaux Préparatoires of the Crime of Aggression, Cambridge University Press, Cambridge 2012, pp. 135 y ss.; acerca del acuerdo del 2010 sobre la definición de crimen de agresión Claus Kress/LeOnie von Holtzendorff. "The Kampala Compromise on the Crime of Aggression", Journal of International Criminal Justice, Vol. 8, 2010, pp. 1179 y ss., y en particular, p. 1189 sobre el requisito de liderazgo.

51 Claus KRESS. "Time for Decision: Some Thoughts on the Immediate Future of the Crime of Aggression: A Reply to Andreas Paulus", European Journal of International Law, Vol. 20, 2009, pp. 1133 y ss. 
Este modelo interestatal sugiere la manera como este privilegio de combatiente puede extenderse a los rebeldes mientras mantiene una poderosa barrera que impide, en principio, el alzamiento en armas. Un Derecho internacional reformado sobre el conflicto armado no internacional puede reposar en dos pilares similares a los de su contraparte interestatal: este puede comprender un privilegio de combatiente en el ius in bello (interno) y al mismo tiempo prohibir, en un nuevo ius contra bellum internum, que cualquier organización realice un ataque contra el gobierno ${ }^{52}$. Al interior de este modelo, el Derecho internacional (salvo en las intervenciones del Consejo de Seguridad de Naciones Unidas) ya no permanecerá indiferente frente a la explosión de un conflicto armado, pero hará de la prevención de la guerra civil su mayor preocupación ${ }^{53}$. Al interior del modelo de reforma bajo consideración, el monopolio en el uso de la fuerza no se debilitará por la introducción del privilegio de combatientes en el Derecho de los conflictos armados no internacionales; este monopolio, de hecho, se fortalecerá por la introducción de una rebelión armada bajo el Derecho internacional ${ }^{54}$. Esta última prohibición, sin embargo, debe quedar limitada al nivel

52 Para una nueva alusión a este nuevo Derecho internacional del conflicto armado no internacional, ver CLAUS KRESS, arriba nota 38; para una propuesta anterior que apunta en la misma dirección, ver NILS Melzer. "Bolstering the Protection of Civilians in Armed Conflict", en A. CASsese, arriba nota 46, pp. 515 y ss.; sin embargo, en la propuesta de Melzer no es del todo claro, si la insurrección armada contra el gobierno debe ser prohibida directamente en el Derecho internacional bajo la amenaza de un castigo (internacional) Ibídem. 517.

53 Este ensayo se concentra principalmente sobre la violencia interna masiva. Sin embargo, debe considerarse con cuidado la cuestión sobre si el nuevo ius contra bellum internum debe también contemplar el ataque transnacional armado que no proviene de un Estado contra un Estado. Este ataque armado no se encuentra en la actualidad contemplado por la definición de Kampala del crimen de agresión en la medida en que los Estados, acorde con el Derecho consuetudinario internacional se han abstenido de incluir los actos no estatales de agresión en la nueva definición del crimen de agresión bajo el derecho internacional. (ver C. KRESS/L. VON HolTZENDORFF, arriba note 50, p. 1190). No se necesitaría un nuevo ius contra bellum internum para extender a ataques armados no estatales si el Derecho internacional consuetudinario ya contiene el crimen internacional de terrorismo (no terrorismo de Estado). En su último e importante pronunciamiento, Antonio Cassese (como juez presidente y ponente) y sus colegas de la Cámara de Apelaciones del Tribunal Especial para el Líbano sostuvieron que este crimen existe y contempla líderes y miembros de bajo rango de un grupo similar a uno terrorista. (Interlocutory Decision on the Applicable Law: Terrorism, Conspiracy, Homicide, Perpetration and Cumulative Charging, STL-11-01/I, 16 de febrero de 2011, para. 111. Bajo la lex data, sin embargo, este descubrimiento judicial abre un problema considerable (para una crítica fuerte, ver sobre todo, Ben SAul, 'Legislating from A Radical The Hague: The un Special Tribunal for Lebanon Invents an International Crime of Terrorism', Leiden Journal of International Law, Vol. 24, 2011, pp. 677 y ss.) y la definición, como la sugiere el Tribunal del Líbano, es peligrosamente amplia si se entiende como candidata para la criminalización directa bajo el Derecho internacional. Por eso se debe dudar seriamente que el Tribunal del Líbano haya marcado el camino hacia una solución satisfactoria en Derecho (penal) internacional para la violencia transnacional no estatal.

54 Un nuevo ius contra bellum internum, como se considera en el texto anterior, deber ser de naturaleza asimétrica, en la medida en que se destinará solo a los actores no estatales; a la luz del monopolio estatal sobre el uso interno de la fuerza, esta asimetría no tiene alternativa; los límites jurídicos internacionales sobre uso interno de la fuerza por el Estado continuarían fluyendo del Derecho internacional de los derechos humanos y la amenaza de pena continuaría siendo consecuencia, en casos 
de liderazgo de los rebeldes (de acuerdo con la definición del crimen de agresión) ${ }^{55}$, de tal manera que el incentivo que se proporcione al soldado rebelde individual, a través del nuevo privilegio de combatiente, para que luche en el conflicto armado no internacional en el marco del Derecho Internacional Humanitario pueda mantenerse ${ }^{56}$.

Es muy delicada la cuestión acerca de si el ius contra bellum internum puede mantenerse sin excepción. Se debe enfatizar que, en consideración al sufrimiento humano en el conflicto armado no internacional (y esto incluye sobre todo el sufrimiento de aquellos que no participan en la lucha), existen razones imperativas para prohibir no solo un cambio por la fuerza de un régimen pro democrático, sino también el alzamiento en armas contra un gobierno que no actúa en (completa) conformidad con los estándares del Derecho Internacional Humanitario. ¿Qué ocurre, sin embargo, si la política interna se vuelve completamente cancerosa, de tal modo que un genocidio o un crimen contra la humanidad de asesinato o exterminio, orquestado por un gobierno, resultan inminentes? A la luz de este escenario, se presenta un fuerte caso de limitación extrema del ius ad bellum internum, que es un derecho de autodefensa de la población civil bajo ataques letales, "hasta tanto el Consejo de Seguridad de Naciones Unidas haya tomado las medidas necesarias para mantener la paz y la seguridad" 57 . Hasta el momento, este ius ad bellum internum solo ha recibido atención académica extremadamente limitada ${ }^{58}$. El problema subyacente

de los más escandalosos abusos, a instancias de agentes estatales, de la criminalización del genocidio y de los crímenes contra la humanidad.

55 Aplicando la lógica subyacente Art. 25 (3) bis del Estatuto de la Corte Penal Internacional mutatis mutandis (acerca de esto, ver C. KRESS and L. VON HOLTZENDORFF, arriba nota 50, p. 1189), los rebeldes de bajo nivel no serían responsables como auxiliares o cómplices.

56 La propuesta de Nils Melzers acerca de 'Fortalecer la Protección de los Civiles en el conflicto armado, en A. CASSESE arriba nota 46, p. 517, no provee una distinción legal entre el nivel de liderazgo y los soldados de bajo nivel. Se puede preguntar si el efecto que él busca lograr a través de la introducción de privilegio de combatiente, no se debilita seriamente por ius contra bellum internum tal y como él lo concibe.

57 En la actualidad, el clásico debate acerca del derecho de los pueblos colonizados a una autodeterminación externa, a la fuerza, no debe llevarse más lejos. En lo que concierna al debate acerca del derecho de un pueblo de secesión remedial a la fuerza por fuera del contexto colonial, se sugiere que este derecho no debe reconocerse. Esto excede el escenario extremo factual como se discute en el texto arriba. Sobre la práctica estatal en este asunto, ver C. KRESS, arriba nota 34, pp. 28 y ss.

58 No obstante, ver FréDÉRIC MÉGret. "Civil Disobedience” and International Law: Sketch for a Theoretical Argument”, Canadian Yearbook of International Law, Vol. 46, 2010, pp. 143 y ss., que identifica el caso de un genocidio inminente como el campo fundamental de aplicación del ius ad bellum internum; en su más reciente contribución al tema, Mégret discute esto último en conjunción con la idea de introducir un privilegio de combatiente en el derecho del conflicto armado no Internacional. En lugar de introducir este privilegio, Mégret sugiere enfocarse decididamente en el lado ad bellum de la moneda y argumenta a favor de un "derecho de los rebeldes a una "amnistía jus post bellum internum" si (y solo si) se han alzado en armas en circunstancias que justifican el ius ad bellum internum; FrÉdÉRIC MÉGRET. "Should Rebels be Amnestied?", en C. STAHn, J. EASTERDAY and J. Iverson (eds.), Jus Post Bellum. Mapping the Normative Foundations, Oxford University Press, Oxford, 2014, pp. 538-539. 
se ha debatido más bien desde la perspectiva de un Estado extranjero interviniente. Ello ha tenido lugar en referencia a la responsabilidad internacional (subsidiaria) de proteger, o de modo más controversial, bajo el encabezado de un (posible) Derecho de los Estados para una forzosa intervención humanitaria ${ }^{59}$. Esto no es particularmente satisfactorio desde la perspectiva de la consistencia interna del Derecho. En un estadio de desarrollo del Derecho internacional, en el que los actores no estatales no necesariamente están relegados a ser beneficiarios indirectos de los derechos de los Estados, la justificación de un derecho excepcional de usar la fuerza en el caso de una inminente catástrofe humanitaria debe estar arraigado en el derecho individual de autodefensa de la población civil que está siendo atacada, y cualquier derecho de un estado extranjero, de acudir a la fuerza en ayuda de la población afectada debe ser concebido como una forma de autodefensa colectiva en beneficio de la población afectada, derivativo de esta autodefensa. En este contexto, es de extremo interés hacer notar que la Liga Árabe, en su declaración de 2013 de la Cumbre de Doha, haya explicado la legalidad de suministrar armamento a la Armada Libre de Siria sobre la base de un Derecho de autodefensa del pueblo de Siria ${ }^{60}$.

En su esencia, un régimen legal completamente simétrico para conflictos armados no internacionales ya se conocía en el siglo XIX, pero en esa época debía ser activado bajo el reconocimiento de beligerancia cuya ocurrencia nunca pudo establecerse. Un Derecho completamente simétrico del conflicto armado no internacional debe ser complementado, sin embargo, por un nuevo Derecho internacional contra la guerra civil. En virtud de este ius contra bellum internun, la prohibición de uso de la fuerza en el Derecho internacional sería extendida a las organizaciones no estatales con capacidad de ejercer violencia masiva. El nuevo ius contra bellum internum puede hacer una contribución a la práctica ya consolidada del Consejo de Seguridad de Naciones Unidas para considerar un conflicto armado no internacional como una amenaza a la paz internacional y la seguridad ${ }^{61}$, y podrá enriquecer el Derecho internacional con la introducción de un concepto discriminatorio de guerra civil hasta ahora desconocido. Por tanto, es teóricamente concebible componer un Derecho no internacional de los conflictos armados no internacionales, que alcance un nuevo estadio de desarrollo bajo el espíritu de Antonio Cassese, y que incluso puede extenderse ligeramente más allá de la última palabra de Cassese en la materia, más allá de

59 Para un análisis de la práctica estatal pertinente, ver C. KREss, arriba nota 34, p. 20 y ss.

60 El pasaje relevante se lee como sigue: “(...) enfatizando en (sic!) el derecho de cada Estado miembro, de acuerdo con su deseo de proporcionar todos los medios para su autodefensa, incluyendo ayuda militar y para soportar la constancia del pueblo sirio y el ejército libre; para acceder al texto completo de la declaración ver, http://arableaguesummit2013.qatarconferences.org/news/news-details-17. html (última visita: 13 de agosto de 2014); sobre la ideas que subyacen a la declaración, ver NiAZ A. SHAн, "The Use of Force under Islamic Law", European Journal of International Law, Vol. 24, 2013, p. 346.

61 Para esta práctica consolidada, Tribunal Penal Internacional para la ex-Yugoslavia, The Prosecutor v Duško Tadić, arriba nota 17, para. 30. 
la distinción entre el ius contra bellum internum asimétrico y el ius in bello interno, reflejando hasta cierto punto el Derecho que rige las relaciones entre Estados. ¿Qué ocurre, sin embargo, con posibles objeciones fundamentales a este desarrollo jurídico y, de ser estas objeciones respondidas, qué ocurre acaso con la puesta en práctica de este nuevo Derecho en beneficio de la humanidad?

\section{¿UNA "UTOPÍA REALIZABLE"?62}

La idea de alcanzar una completa simetría en el Derecho de los conflictos armados no internacionales da razones para hacer una pausa y lanzar, en primer lugar, una nueva mirada a la legitimidad del privilegio de combatientes en el Derecho del conflicto armado internacional. A este respecto, el punto de partida es reconocer que, en la época de prohibición de uso de la fuerza en las relaciones internacionales, la legitimidad de una permisión de usar la fuerza letal para soldado de un estado agresor debe poderse cuestionar. Hace un par de años, Jeff McMahan sometió el privilegio de combatiente para el "guerrero injusto" a una crítica bastante elaborada en términos de filosofía moral. Él argumentó que ni la idea de consentimiento válido ni de una presunción a favor de un inevitable error de Derecho ni la dependencia a una presunta orden superior de hacer la guerra dada por los líderes del Estado agresor constituyen buenas razones para justificar ese privilegio ${ }^{63}$. Es importante prestar atención al argumento de McMahan, pues tiene incluso más peso respecto de un "guerrero injusto" no estatal en un conflicto armado no internacional. Sin embargo, McMahan se ha rehusado explícitamente (mientras añade la advertencia "en el presente") a argumentar en favor de una construcción asimétrica del Derecho internacional de los conflictos armados como una consecuencia de su argumento moral. En ese contexto (entre otras cosas), se refiere al incentivo que emana del privilegio de combatientes para "guerreros injustos" al conformarse con el Derecho de los conflictos armados ${ }^{64}$. En un concienzudo libro recientemente publicado, Frédéric Mégret toma una posición diferente y cuestiona el privilegio de combatiente simétrico en el Derecho del conflicto armado internacional. Esta posición está (entre otras cosas) basada en la incredulidad de que el privilegio de combatientes de hecho opere como un incenti-

62 La expresión alude al título del libro referido arriba en la nota 46, como fue editado por Antonio Cassese.

63 JefF McMahan. Killing in War, Oxford University Press, Oxford, 2009, pp. 38 y ss.

64 JefF McMahan. Ibídem., p. 190/1: "Los combatientes injustos que temen al castigo al final de la guerra pueden estar más reacios a rendirse, prefiriendo continuar la lucha con una baja probabilidad de victoria que rendirse con una alta probabilidad de ser castigados. (...) Ellos pueden tener el razonamiento de que si se confrontan con un castigo masivo en el caso de la derrota, tienen poco que perder si abandonan todas las restricciones que puedan tener en su esfuerzo de ganar. Por ejemplo, ellos pueden tener el razonamiento de que si son derrotados y los acusadores de modo poco probable conocen de sus actos individuales, ninguno tiene nada que perder, pero algo que ganar en la comisión de crímenes atroces, que aumentará sus posibilidades de victoria y la inmunidad del castigo". Para una línea similar de razonamiento, ver Nigel Biggar, In Defence of War, Oxford University Press, Oxford, 2013, p. 196. 
vo de cumplimiento. Mégret denuncia la opinión ampliamente sostenida, como si esta no se basara más que en asunciones psicológicas de personas comunes ${ }^{65}$. Estas dudas deben ser tratadas con la seriedad del caso, además de que hacen un llamado para otra ronda de reflexión acerca del incentivo de potencial cumplimiento que el privilegio de combatiente pueda tener para el "guerrero injusto", en relación con el Derecho vigente del conflicto armado internacional y el posible futuro del Derecho del conflicto armado no internacional. En lo que tiene que ver con el Derecho del conflicto armado no internacional, que es el corazón de este ensayo, hay algo que queda claro: la introducción del privilegio de combatiente para luchadores no estatales en un conflicto armado no internacional necesita de la posibilidad realista de poder mejorar el cumplimiento del Derecho Internacional Humanitario por las partes no estatales del conflicto.

La imposibilidad de justificar esta perspectiva no va a eliminar el debate acerca de un nuevo ius contra bellum internum. Es cierto que la idea de esta nueva ley ha sido presentada aquí como un ius in bello interno completamente simétrico. Sin embargo, se debe enfatizar que un nuevo ius contra bellum internum (incluyendo un ius ad bellum internum excepcional en caso de una inminente catástrofe humanitaria) debe ser objeto de debate en cualquier evento. En otras palabras: un movimiento hacia una simetría completa en el Derecho de los conflictos armados no internacionales depende de la introducción de un nuevo ius contra bellum internum. Sin embargo, esta afirmación no necesariamente es verdadera si se invierten sus componentes.

No obstante, la idea de un nuevo ius contra bellum internum abre paso para importantes preguntas. En primer lugar, se debe pensar con cuidado la cuestión sobre si una formulación de la nueva prohibición internacional (bajo la amenaza de un castigo internacional) así como excepción limitada es posible, lo cual resulta tanto sustancialmente satisfactorio como suficientemente preciso ${ }^{66}$. También es necesaria la confirmación acerca de si un nivel de liderazgo puede identificarse en la mayoría de las instancias de un (emergente) conflicto armado, de tal manera que la nueva regulación internacional pueda de hecho funcionar como la pretendida barrera legal contra desencadenamiento de las guerras civiles. Más aún, el funcionamiento práctico de este nuevo régimen legal bajo consideración debe ser puesto en una seria duda si el estallido de un conflicto armado no internacional ha sido precedido por una dinámica de escalamiento que no puede desenredarse con una claridad suficiente que permita de modo confiable atribuir responsabilidades. Desafortunadamente, la situación de Siria (desde el 2012) y de Ucrania (2013/2014) nos proporcionan

65 Frédéric MÉGret. “Should Rebels be Amnestied?”, en C. Stahn,J. S. EAsterday and J. Iverson (eds.), arriba nota 58, pp. 538-539.

66 Para la cuestión sobre si se debe o no incluir la violencia transnacional no estatal, ver arriba nota 53. Además, para respetar debidamente la esfera doméstica de jurisdicción, el ámbito de aplicación de la nueva regla internacional tendría que limitarse a una violencia estatal que alcance el umbral del conflicto armado no internacional. 
ejemplos frescos de este tipo de dinámica. Con todo, un nuevo desafío resulta del hecho de que hay constituciones nacionales que conceden el derecho a la resistencia armada bajo ciertas condiciones ${ }^{67}$. Allí habría una necesidad de armonizar los dos niveles de regulación jurídica. Finalmente, no cabe duda de que una introducción de ius contra bellum internum constituiría una reforma jurídica muy ambiciosa. Uno podría preguntarse si ello está demasiado lejos del Derecho internacional, por lo menos para el tiempo presente.

De cara a estas importantes y hasta el momento escasamente discutidas cuestiones, el modelo de reforma para el Derecho internacional de conflictos armados no internacionales no se presenta aquí para su inmediata adopción. Por el contrario, este modelo se presenta con espíritu académico para debatir si esta puede ser una "utopia realizable" en los términos de Antonio Cassese. Será un debate multifacético y estimulante. Con todo, las guerras civiles son uno de los peores males de nuestro tiempo. Una mejor aunque modesta contribución al Derecho internacional que permite lidiar con este mal en sus raíces, justificaría, por tanto, el mayor y más demandante esfuerzo intelectual.

\section{BIBLIOGRAFÍA}

Andreas Paulus/Mindia Vashakmadze. "Asymmetrical War and the Notion of Armed Conflict: A Tentative Conceptualization", International Review of the Red Cross, Vol. 91, 2009.

Anthony Cullen. The Concept of Non-International Armed Conflict in International Humanitarian Law, Cambridge University Press, Cambridge, 2010.

Antonio CASSESE (ed.). Current Problems of International Law. Essays on U.N. Law and on the Law of Armed Conflict, Dott. A. Giuffrè Editore, Milan, 1975.

Antonio CAssese (ed.). The New Humanitarian Law of Armed Conflict, Editoriale Scientifica S.r.l., Naples, 1979.

Antonio Cassese. "Should Rebels be Treated as Criminals? Some Modest Proposals for Rendering Internal Armed Conflicts Less Inhumane”, en ANTONIO CASSESE (ed.). Realizing Utopia. The Future of International Law, Oxford University Press, Oxford, 2012.

67 Por ejemplo, ver Art. 20 (4) de la Ley Fundamental Alemana. 
Auswärtiges Amt/Deutsches Rotes Kreuz/Bundesministerium der Verteidigung (eds), Dokumente zum Humanitären Völkerrecht, 2nd ed., Academia Verlag, Sankt Augustin, 2012.

Ben SAul. 'Legislating from A Radical The Hague: The UN Special Tribunal for Lebanon Invents an International Crime of Terrorism', Leiden Journal of International Law, Vol. 24, 2011.

CHRISTOPHE SWINARSKI (ed.). Études et essais sur le droit international humanitaire et sur les principes de la Croix-Rouge en l'honneur de Jean Pictet, ICRC/Martinus Nijhoff Publishers, Geneva/The Hague, 1984.

Claus Kress, "Major Post-Westphalian Shifts and Some Important Neo-Westphalian Hesitations in the State Practice on the International Law on the Use of Force", Journal on the Use of Force and International Law, Vol. 1, 2014.

Claus Kress. "Review of Books", British Yearbook of International Law, Vol. 83, 2012

Claus Kress. "Some Reflections on the International Legal Framework Governing Transnational Armed Conflict", Journal of Conflict \& Security Law, Vol. 15, 2010.

Claus Kress. "Time for Decision: Some Thoughts on the Immediate Future of the Crime of Aggression: A Reply to Andreas Paulus", European Journal of International Law, Vol. 20, 2009.

Claus Kress. "War Crimes Committed in Non-International Armed Conflict and the Emerging System of International Criminal Justice”, Israel Yearbook on Human Rights, Vol. 30, 2000.

Claus Kress/Leonie von HoltzendorfF. "The Kampala Compromise on the Crime of Aggression”, Journal of International Criminal Justice, Vol. 8, 2010.

EMILY CRAWFORD. The Treatment of Combatants and Insurgents under the Law of Armed Conflict, Oxford University Press, Oxford, 2010.

FRÉDÉRIC MÉGRET. “Civil Disobedience” and International Law: Sketch for a Theoretical Argument”, Canadian Yearbook of International Law, Vol. 46, 2010.

Frédéric MÉGret. “Should Rebels be Amnestied?”, en C. Stahn, J. EASterday and J. Iverson (eds.), Jus Post Bellum. Mapping the Normative Foundations, Oxford University Press, Oxford, 2014. 
JeAn D’Asprémont y Jérôme De Hemptinne. Droit international humanitaire, Editions A. Pedone, Paris, 2012.

Jean S. Pictet. The Geneva Conventions of 12 August 1949. Commentary: IV Geneva Convention Relative to the Protection of Civilian Persons in Time of War, Geneva, 1958.

Jean-Henry Dunant. Un souvenir de Solférino, Cassel, London, 1947.

Jean-Marie Henckaerts and Louise Doswald-Beck (eds.). Customary International Humanitarian Law, Vol. I: Rules. Vol. II: Practice, Cambridge University Press, Cambridge, 2005.

JefF McMahan. Killing in War, Oxford University Press, Oxford, 2009.

Kenneth Watkin. "Opportunity Lost: Organized Armed Groups and the ICRC 'Direct Participation in Hostilities' Interpretive Guidance”, New York University Journal of International Law and Politics, Vol. 42, 2010.

LINDSAY MOIR. The Law of Internal Armed Conflict, Cambridge University Press, 2002

Mary Ellen O'Connell. "Combatants and Combat Zone", University of Richmond Law Review, Vol. 43, 2008-2009.

Mary Ellen O'Connell. "Historical Development and Legal Basis", en DieTer FLECK (ed.), The Handbook of International Humanitarian Law, 3rd ed., Oxford University Press, Oxford, 2013.

NiAZ A. SHAн. "The Use of Force under Islamic Law", European Journal of International Law, Vol. 24, 2013.

Nigel Biggar. In Defence of War, Oxford University Press, Oxford, 2013.

Nils Melzer. "Keeping the Balance Between Military Necessity and Humanity: A Response to Four Critiques of the ICRC's Interpretive Guidance on the Notion of Direct Participation in Hostilities", New York University Journal of International Law and Politics, Vol. 42, 2010.

Noam Lubell y Nathan Derejko. “A Global Battlefied. Drones and the Geographical Scope of Armed Conflict”, Journal of International Criminal Justice, Vol. 11, 2013.

NoAm Lubell. Extraterritorial Use of Force Against Non-State Actors, Oxford University Press, Oxford, 2010. 
ReINHARD MerKel. "Die 'kollaterale' Tötung von Zivilisten im Krieg. Rechtsethische Grundlagen und Grenzen einer prekären Erlaubnis des humanitären Völkerrechts“, Juristen Zeitung, Vol. 67, 2012.

Robin GeIss. “Military Necessity: A Fundamental 'Principle' Fallen Into Oblivion”, en HÉlène Ruiz Fabri, Rüdiger Wolfrum, Jana Gogolin (eds.), Select Proceedings of the European Society of International Law, Hart Publishing, Oxford and Portland, 2008.

SANDESH SivaKuRAman. The Law of International Armed Conflict, Oxford University Press, 2012.

Stefan Barriga/Claus Kress (eds.). The Travaux Préparatoires of the Crime of Aggression, Cambridge University Press, Cambridge, 2012.

The Problem of the Revision of the Law of War", British Year Book of International Law, Vol. 29, 1952.

Theodor Meron. “The Humanization of Humanitarian Law”, American Journal of International Law, Vol. 94, 2000.

Thomas Buergenthal. "Human Rights", en Rüdiger Wolfrum (ed.), The Max Planck Encyclopedia of Public International Law. Vol. IV, Oxford University Press, 2012.

W. HAYs PARKS. "Part IX of the ICRC 'Direct Participation Study': No Mandate, No Expertise, and Legally Incorrect", New York University Journal of International Law and Politics, Vol. 42, 2010.

YoRAm Dinstein. The Conduct of Hostilities under the Law of International Armed Conflict, $2^{\text {a }}$ ed., Cambridge University Press, Cambridge, 2010. 